\title{
A Multiple Attribute Decision Method Based on Fuzzy Flexibility Optimum Seeking Theory \\ Qin Wang ${ }^{1, a}$ and Liang $\mathrm{Ma}^{1, \mathrm{~b}}$
}

\author{
Dept. of Surface Ship Command, Dalian Naval Academy, Dalian 116018, China \\ aalbert77618@163.com, ${ }^{\mathrm{b}}$ maliang2014@tom.com
}

Keywords: Multiple attributes decision; Fuzzy optimum seeking; Non-Normal fuzzy number; Fuzzy distance

\begin{abstract}
Normally, Fuzzy decision can show the faintness of the decision resultant, but can't show the faintness of the decision maker. This paper will introduce the concept of the non-normal fuzzy number and fuzzy distance, and then improve on the multiple attribute decision method. This not only can present the faintness of the decision resultant, but also can present the belief of the decision maker and the degree of the group decision.
\end{abstract}

\section{Introduction}

In 1988, Professor Shouyu Chen advanced the concept of comparative subject in Dalian university of technology, and then he constituted the model of fuzzy optimization. In 2003, Doctor Xiancheng Huang got more extending about the concept. These theories are based on the fuzzy denotation for the decision precept. But practicality, not only the object of decision precept is fuzzy, the man who makes decision also have the illegibility. The illegibility of decider incarnates two sides: one is the confidence about decision; another is the degree of the group of decision-making. This paper uses non-normal fuzzy number to denote the decided conclusion, not only incarnate the denotation of object of decision precept, but can denote the two characters of decider.

The research content of this paper includes two aspects: fuzzy distance of non-normal number and multi-attribute fuzzy flexibility optimum seeking model.

\section{Non-Normal Fuzzy Number and Fuzzy Distance}

Normal Fuzzy Number. Normal fuzzy number commonly is for short to fuzzy number. On the assumption, $\tilde{A}$ is a subset of real number set of $R$, the membership function is $\mu_{\tilde{A}}$. Then we can take $\tilde{A}$ as a fuzzy number, if it can fulfill nether condition.

(1) for arbitrarily $\alpha \in(0,1)$, the cut set $\alpha$ of $\tilde{A}$ always is convex set;

(2) $\mu_{\tilde{A}}$ is a upper semi-continuous function;

(3) $\tilde{A}$ is the bounded set of $R$;

Non-normal fuzzy number. The concept of non-normal number must fulfill nether conditions.

(1) 'non-normal': $\exists x \in R, 0<\max \left\{\mu_{\tilde{A}}(x)\right\}<1$;

(2) convex set;

When $\max \left\{\mu_{\tilde{A}}(x)\right\}=1$, the non-normal fuzzy number is the normal fuzzy number. Fig. 1 denotes a normal fuzzy number (solid line) and non-normal fuzzy number (broken line) which is close to reaching 5 . 


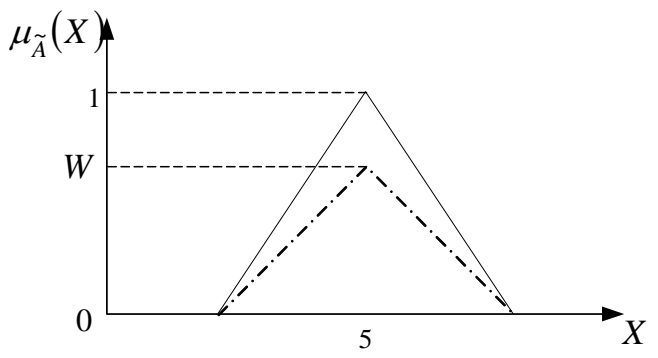

Figure 1. The fuzzy number which is closed to 5

We have many methods to express fuzzy number. The fuzzy number of Fig. 2 can be expressed as $\widetilde{A}=(a, b, c, d ; W)[1]$.It's altitude $W$ belongs $(0,1)$.

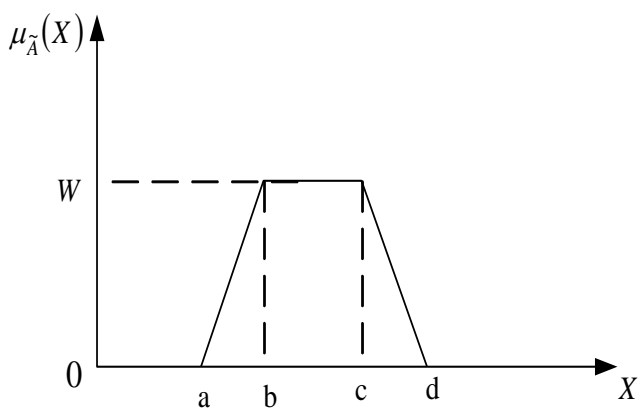

Figure 2. Trapezoidal fuzzy number

When $b=c$, the fuzzy number of Fig. 2 is triangular fuzzy number. When $a=b$ and $c=d$, it is rectangle fuzzy number. On the assumption, $\widetilde{A}=\left(a_{1}, a_{2}, a_{3}, a_{4} ; W_{1}\right)$ and $\widetilde{B}=\left(b_{1}, b_{2}, b_{3}, b_{4} ; W_{2}\right)$, We can confirm the operation arithmetic of non-normal fuzzy number [2].

Addition: $\tilde{A} \oplus \tilde{B}=\left(a_{1}+b_{1}, a_{2}+b_{2}, a_{3}+b_{3}, a_{4}+b_{4}, \min \left(W_{1}, W_{2}\right)\right)$

Subtraction: $\tilde{A}-\widetilde{B}=\left(a_{1}-b_{1}, a_{2}-b_{2}, a_{3}-b_{3}, a_{4}-b_{4}, \min \left(W_{1}, W_{2}\right)\right)$

Multiplication: $\tilde{A} \otimes \widetilde{B}=\left(a_{1} \otimes b_{1}, a_{2} \otimes b_{2}, a_{3} \otimes b_{3}, a_{4} \otimes b_{4}, \min \left(W_{1}, W_{2}\right)\right)$

Division: $\tilde{A} \div \tilde{B}=\left(a_{1} / b_{1}, a_{2} / b_{2}, a_{3} / b_{3}, a_{4} / b_{4}, \min \left(W_{1}, W_{2}\right)\right)$

Non-normal fuzzy distance. In order to measure the difference between two fuzzy numbers, we can introduce the distance concept. About the real number field $U$, the Minkowski distance between fuzzy number $\tilde{M}$ and $\tilde{N}$ can be expressed as Eq.5 [3].

$$
d_{p}(\tilde{M}, \tilde{N})=\left\{\int_{x \in U}\left[\mu_{\tilde{M}}(x)-\mu_{\tilde{N}}(x)\right]^{p} d x\right\}^{\frac{1}{p}}
$$

Of which, $p$ is called distance parameter, when we get different value of $p$, we can get different expression format of fuzzy distance.

When $p=1$, we take it as Hamming distance.

$$
d_{1}(\tilde{M}, \tilde{N})=\int_{x \in U}\left[\mu_{\tilde{M}}(x)-\mu_{\tilde{N}}(x)\right] d x
$$

When $p=2$, we take it as Euclidean distance. 


$$
d_{2}(\tilde{M}, \tilde{N})=\left\{\int_{x \in U}\left[\mu_{\tilde{M}}(x)-\mu_{\tilde{N}}(x)\right]^{2} d x\right\}^{\frac{1}{2}}
$$

When $p=\infty$, we take it as Chebyshev distance.

$$
d_{\infty}(\tilde{M}, \tilde{N})=\max _{x \in U}\left|\mu_{\tilde{M}}(x)-\mu_{\tilde{N}}(x)\right|
$$

According Eq.1, Eq.2, Eq.3, Eq.4, we can expand Eq.5, Eq.6, Eq.7, and Eq.8 to the expression of non-normal fuzzy number distance.

On the assumption, $\tilde{M}$ and $\tilde{N}$ are the non-normal fuzzy numbers of real number field $U$, the altitude of their membership function respectively is $\lambda_{M}$ and $\lambda_{N}$, so, the Minkowski distance between fuzzy number $\tilde{M}$ and $\tilde{N}$ can be expressed as Eq.9.

$d_{p}(\tilde{M}, \tilde{N})=\min \left(\lambda_{M}, \lambda_{N}\right)\left\{\int_{x \in U}\left[\mu_{\tilde{M}}(x)-\mu_{\tilde{N}}(x)\right]^{p} d x\right\}^{\frac{1}{p}}$

When we use triangular fuzzy number and trapezoidal fuzzy number to express decision resultant. Then, for non-normal fuzzy number $\tilde{A}=\left(a_{1}, a_{2}, a_{3} ; \lambda_{1}\right)$ and $\tilde{B}=\left(b_{1}, b_{2}, b_{3} ; \lambda_{2}\right)$, their Minkowski distance can be expressed as Eq.10.

$$
d_{p}(\tilde{A}, \tilde{B})= \begin{cases}\min \left(\lambda_{1}, \lambda_{2}\right)\left(\frac{1}{3} \sum_{i=1}^{3}\left|a_{i}-b_{i}\right|^{p}\right)^{\frac{1}{p}} & 1 \leq p<\infty \\ \max _{i}\left|a_{i}-b_{i}\right| \cdot \min \left(\lambda_{1}, \lambda_{2}\right) & p=\infty\end{cases}
$$

For trapezoidal fuzzy number $\tilde{A}=\left(a_{1}, a_{2}, a_{3}, a_{4} ; \lambda_{1}\right)$ and $\widetilde{B}=\left(b_{1}, b_{2}, b_{3}, b_{4} ; \lambda_{2}\right)$, their Minkowski distance can be expressed as Eq.11.

$$
d_{p}(\tilde{A}, \tilde{B})= \begin{cases}\min \left(\lambda_{1}, \lambda_{2}\right)\left(\frac{1}{4} \sum_{i=1}^{4}\left|a_{i}-b_{i}\right|^{p}\right)^{\frac{1}{p}} & 1 \leq p<\infty \\ \max _{i}\left|a_{i}-b_{i}\right| \cdot \min \left(\lambda_{1}, \lambda_{2}\right) & p=\infty\end{cases}
$$

\section{Fuzzy Flexibility Optimum Seeking}

On the assumption that decision question's relative membership degree matrix $\tilde{R}$ and attribute weight vector $\tilde{w}$ are given.

$$
\begin{aligned}
\tilde{R} & =\left(\begin{array}{cccc}
\tilde{r}_{11} & \tilde{r}_{12} & \cdots & \tilde{r}_{1 n} \\
\tilde{r}_{21} & \tilde{r}_{22} & \cdots & \tilde{r}_{2 n} \\
\vdots & \vdots & \vdots & \vdots \\
\tilde{r}_{m 1} & \tilde{r}_{m 2} & \cdots & \tilde{r}_{m n}
\end{array}\right)=\left(\tilde{r}_{i j}\right)_{m \times n} \\
\tilde{w} & =\left(\tilde{w}_{1}, \tilde{w}_{2}, \cdots, \tilde{w}_{n}\right)
\end{aligned}
$$


Of which, $i, j, m, n$ are positive integer, $m$ expresses the number of program, $n$ expresses the number of attribute index of program.

$\tilde{w}_{j}$ is the weight value of number- $j$, it is fuzzy number.

$\tilde{r}_{i j}$ is the relative membership degree value of attribute index number- $j$ of number- $i$ program, it can be non-normal fuzzy number or normal fuzzy number, it's value can be given by the decision matrix of the question[4].

On the assumption, $\lambda_{i j}$ is the membership function altitude of $\tilde{r}_{i j}$, when the normal fuzzy number $\lambda_{i j}=1$ is in the relative membership degree matrix , $\tilde{r}_{i j}$ can express three properties.

(1) The fuzzy expression about the decision resultant;

(2) Confidence level of decision makers for their participation in decision-making;

(3) When they make group decisions, show that the degree of decision-making team consensus on a subject;

According to general fuzzy optimization model, we can extend it to general fuzzy flexibility under the circumstance of the optimization model.

$$
\mu_{i}=\frac{1}{1+\left\{\frac{\sum_{j=1}^{n}\left[d\left(\tilde{w}_{j} \tilde{r}_{i j}, \tilde{w}_{j}\right)\right]^{q}}{\sum_{j=1}^{n}\left[d\left(\tilde{w}_{j} \tilde{r}_{i j}, 0\right)\right]^{q}}\right\}^{\frac{2}{q}}}
$$

In Eq.12: $\mu_{i}$ says to the $i$ solution relative to the optimal weights. $q$ is the distance parameter, when $q=1$, as Hamming distance, when $q=2$, as Euclidean distance, when $q=\infty$, as Chebyshev distance. $d\left(\widetilde{w}_{j} \tilde{r}_{i j}, \tilde{w}_{j}\right)$ is space form optimal distance of the $i$ solution. $d\left(\tilde{w}_{j} \tilde{r}_{i j}, 0\right)$ is space from carrier. Their value can be calculated by Eq.1 to Eq.2 and Eq.9.

On the assumption, $\tilde{w}_{j}=\left(a_{1 j}, a_{2 j}, a_{3 j} ; \zeta_{j}\right), \tilde{r}_{i j}=\left(b_{1 i j}, b_{2 i j}, b_{3 i j}, \lambda_{i j}\right)$, the Eq.12 can be converted to Eq.13.

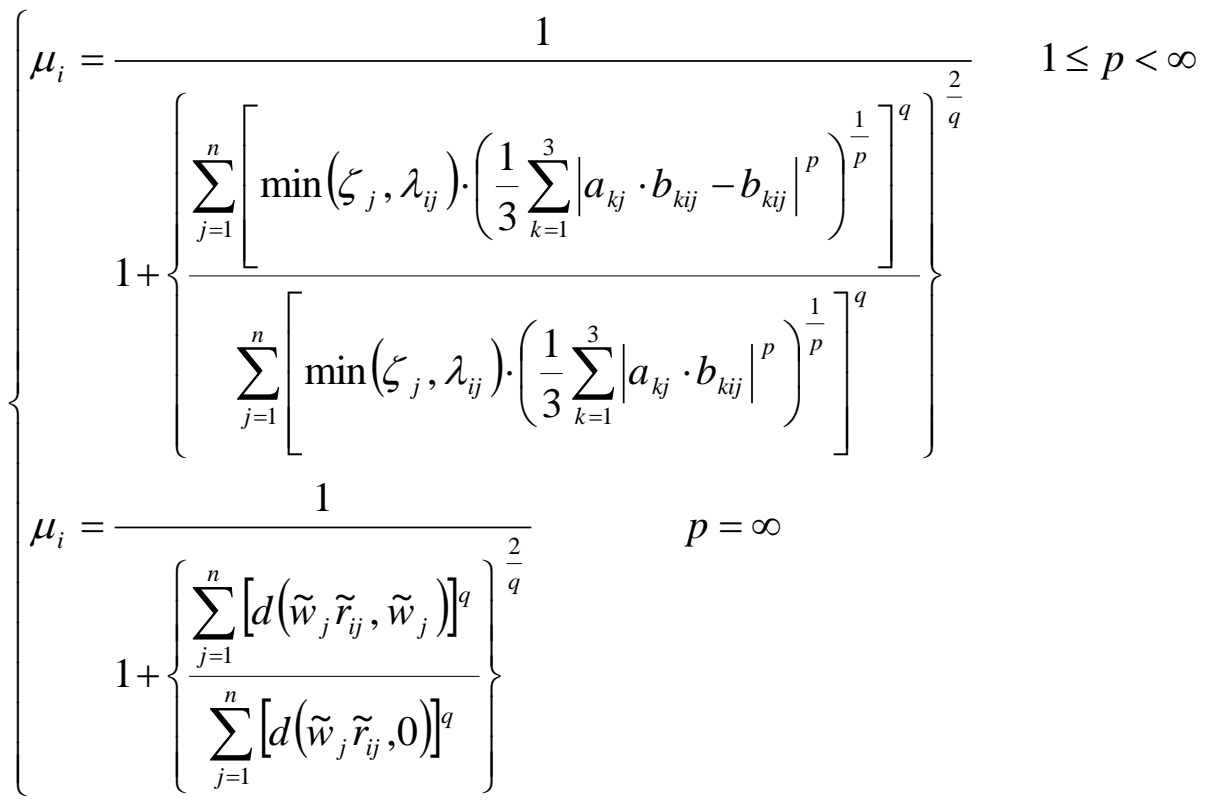


The same model can be concluded that $\tilde{w}_{j}$ and $\tilde{r}_{i j}$ are Trapezoidal non-normal fuzzy number.

\section{The Example}

A certain river basin administrative agencies achieve to a more effective development, utilization, protection and management of water resources. Considering specifying river basin development plan for a long time, after a preliminary screening, finally three development planning was determined, for example $A_{1}, A_{2}$ and $A_{3}$. Now on the basis of the following five goals the decision makers will make their optimization.

(1) The coordinated development degree with the natural environment and humane environment, this paper uses $o_{1}$ to express;

(2) Comparing between income and investment, this paper uses $O_{2}$ to express;

(3) The degree of sustainable development, this paper uses $o_{3}$ to express;

(4) The economic effect for the promotion of regional, this paper uses $o_{4}$ to express;

(5) Coordinated development of ecological system, this paper uses $o_{5}$ to express.

Of which, the binary comparison results of decision makers for the importance of targets: $o_{1} \approx o_{2} \approx o_{3} \succ o_{4} \succ o_{5}, \quad o_{1}$ to $o_{4}$ is more important, $o_{1}$ is a little important than $o_{5}$.

First calculate the normal fuzzy number plan optimization results[5].

$$
\tilde{\omega}=(0.214,0.214,0.214,0.182,0.176)
$$

$$
\begin{aligned}
& \begin{array}{lll}
A_{1} & A_{2} & A_{3}
\end{array}
\end{aligned}
$$

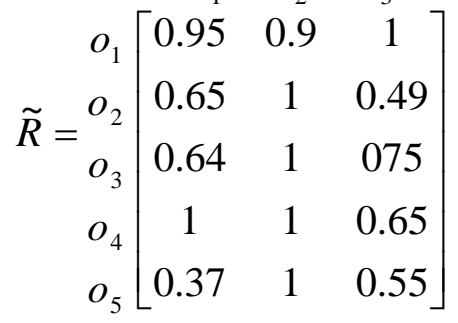

According to Eq.12, and get the value of $p=1, q=2$, we can get the optimal size vector of solution.

$u=(0.843,0.995,0.817)$

According to the principle of maximum membership degree, we can get the good order of solution: $A_{2} \succ A_{1} \succ A_{3}$.

On the assumption, non-normal triangular fuzzy number is used to program on the target set for binary comparison, then we can get the target weight vector $\tilde{w}$ and optimal relative matrix $\tilde{R}$.

$$
\tilde{\omega}=\left[\begin{array}{c}
(0.2,0.214,0.228 ; 0.6) \\
(0.2,0.214,0.228 ; 0.4) \\
(0.2,0.214,0.228 ; 0.5) \\
(0.17,0.182,0.194 ; 0.7) \\
(0.164,0.176,0.188 ; 0,8)
\end{array}\right]^{T}
$$




$$
\begin{aligned}
& \begin{array}{lll}
A_{1} & A_{2} & A_{3}
\end{array} \\
& \tilde{R}=\begin{array}{c}
o_{1} \\
o_{2} \\
o_{3} \\
o_{4} \\
o_{5}
\end{array}\left[\begin{array}{cccc}
(0.92,0.95,0.98 ; 0.6) & (0.88,0.9,0.92,0.65) & (0.96,1,1.04 ; 0.8) \\
(0.63,0.65,0.67 ; 0.5) & (0.98,1,1.02 ; 0.8) & (0.47,0.49,0.51 ; 0.6) \\
(0.62,0.64,0.66 ; 0.5) & (0.98,1,1.02 ; 0.8) & (0,73,0.75,0.77 ; 0.6) \\
(0.98,1,1.02 ; 0.8) & (0.98,1,1.02 ; 0.8) & (0.63,0.65,0.67 ; 0.7) \\
(0.35,0.37,0.39,0.4) & (0.98,1,1.02 ; 0.8) & (0.53,0.55,0.57,0.5)
\end{array}\right]
\end{aligned}
$$

According to Eq.13, and get the value of $p=1, q=2$, we can get the optimal size vector of solution.

$u=(0.886,0.969,0.893)$

According to the principle of maximum membership degree, we can get the good order of solution: $A_{2} \succ A_{3} \succ A_{1}$.

By the above two results, we can see that the non-normal fuzzy number can show its three characteristics.

\section{References}

[1] S. H. Chen: Operations on fuzzy numbers with function principle[J], Tamkang Journal of Management Sciences, vol. 6 (1985) No. 1, p.13-25.

[2] Zeshui Xu: Based on fuzzy language evaluation and operator method of multiple attribute group decision making[J]. Systems engineering, Vol. 20 (2002) No. 5. (In Chinese)

[3] R.R.Yager and D.P.Filev: Induced ordered weighted averaging operators[J] . IEEE Transactions on Systems, Man, and Cybernetics-Part B: Cybernetics, vol. 29 (1999) No. 2, p. 141-150.

[4] S. J. Chen and S. M. Chen: Handling Multicriteria Fuzzy Decision making Problems Using FN-IOWA Operators[J] . Proceedings of the Eleventh International Conference on Information Management, Kaohsiung, Taiwan, R. O. C., 2000

[5] Xiancheng Huang: Fuzzy multi-objective decision making theory and application[D]. Dissertation. Dalian university of technology, 2003. (In Chinese) 